\title{
A CONDUCTIVE COOLING MODEL FOR CONFINED SOLAR FLARE PLASMA*
}

\author{
L. W. ACTON and W. T. ZAUMEN \\ Lockheed Palo Alto Research Laboratory, Palo Alto, Calif., U.S.A.
}

\begin{abstract}
Solar Phys.). The evolution of the X-ray emission from hot solar plasmas for which radiative cooling is insignificant is computed on the basis of a simple conduction model. This model considers a hot and a cool region separated by a sharp boundary. The plasma is assumed to be confined within a vertical column of constant cross-sectional area with the hot X-ray emitting region lying above the cool region, and both being in hydrostatic equilibrium. Because the electron temperature is high and thermal conductivity varies at $T^{5 / 2}$ the hot region remains approximately isothermal during the cooling process. Cooling then proceeds by raising the temperature of the cool material at the interface thereby moving the boundary into the cool region. The emission measure, E.M. $\equiv \int N_{e}^{2} d V$, of the hot volume therefore increases as the cooling progresses. For this model, the emission measure may be expressed as a function of temperature, $T$, by $\{$ E.M. $\}=\left\{\right.$ E.M. $\left.{ }_{0}\right\}\left(T_{0} / T\right)\left[\left(T_{0}-T_{f}\right) /\left(T-T_{f}\right)\right]^{2}$ where E.M. ${ }_{0}$ is the emission measure when the hot region is at temperature $T_{0}$, and $T_{f}$ is the temperature of the cool region. This expression is shown to satisfactorily describe observations of small fast-decaying flares. Larger, slower events tend to show a decreasing emission measure during late decay phases and cannot be fitted with this model.
\end{abstract}

* This research has been carried out under support of NASA Contract NASw-2316 and by the Lockheed Independent Research Program. 